\title{
Fiabilidad y validez de constructo de la traducción al valenciano/catalán del Cuestionario para la Evaluación del Síndrome de Quemarse por el trabajo (CESOT) en docentes no universitarios
}

\section{Reliability and construct validity of the Valencian/ Catalonian translation of the "Spanish Burnout Inventory" (SBI) in a sample of non-university teachers}

Fecha de recepción: 10-10-2020

Fecha de aceptación: 17-05-2021
José Luis Llorca-Rubio Instituto Valenciano de Seguridad e Higiene en el Trabajo. España

Marta Llorca-Pellicer,

Unidad de Investigación Psicosocial de la Conducta Organizacional (UNIPSICO), Universitat de València. España

Pedro R. Gil-Monte

Unidad de Investigación Psicosocial de la Conducta Organizacional (UNIPSICO), Universitat de València. España

\section{resumen/ahstract:}

El objetivo de este estudio fue evaluar la fiabilidad y validez de constructo del «Cuestionario para la Evaluación del Síndrome de Quemarse por el Trabajo» (CESQT) en una muestra de profesores no universitarios de la provincia de Valencia (España). La muestra la formaron 1359 docentes (67.2\% mujeres). El cuestionario consta de 20 ítems distribuido en cuatro escalas: Ilusión por el trabajo (5 ítems), Desgaste psíquico (4 ítems), Indolencia (6 ítems) y Culpa (5 ítems). Las propiedades psicométricas fueron examinadas mediante los siguientes análisis: Análisis factorial confirmatorio (AFC) y fiabilidad (alfa de Cronbach). Todos los ítems presentaron valores estadísticos y psicométricos adecuados así como las escalas. El modelo de cuatro factores que reproduce la estructura original del cuestionario presentó un ajuste adecuado: $\mathrm{AGFI}=.926, \mathrm{NNFI}=.919, \mathrm{CFI}=.930, \mathrm{RMSEA}=.053_{(.050-057)}$. El valor del alfa de Cronbach resultó aceptable para las cuatro escalas del instrumento. Estos resultados contribuyen a la validación psicométrica del cuestionario, permitiendo concluir que el CESOT, en valenciano/catalán resulta un instrumento fiable y válido para evaluar el SQT en personal docente no universitario.

The aim of this study was to evaluate the reliability and construct validity of the Valencian/Catalonian version of the Spanish Burnout Inventory (SBI) in a sample of non-university teachers from the Valencia province (Spain). The sample consisted of 1359 teachers (67.2\% were women). This instrument contains 20 items distributed into four dimensions: Enthusiasm toward the job (5 items), Psychological exhaustion (4 items), Indolence (6 items) and Guilt (5 items). The psychometric properties were examined through the following analyses: confirmatory factor analysis (CFA) and reliability (Cronbach's alpha). All items achieved appropriate values for both statistical and psychometric parameters, just as the four scales did. The four-factor model consistent with the Spanish original obtained a good data model fit: $\mathrm{AGFI}=.926, \mathrm{NNFI}=.919, \mathrm{CFI}=.930, \mathrm{RMSEA}=.053_{(.050 .057) .}$ The Cronbach's alpha value was acceptable for the four scales of the instrument. These results contribute to the psychometric validation of the questionnaire, allowing to conclude that the CESOT, in Valencian / Catalan, is a reliable and valid instrument for evaluating the SOT in non-university teaching personnel.

\section{palabras clave/keywords:}

síndrome de quemarse por el trabajo, estrés laboral, docentes, análisis factorial confirmatorio, CESQT Burnout, job stress, teachers, confirmatory factor analysis, Spanish Burnout Inventory (SBI). 


\section{Introducción}

El estrés laboral está generalizado entre los profesionales docentes (Travers, 2017), presentando un alto riesgo de experimentar niveles elevados de estrés laboral crónico y problemas derivados de esta exposición, como el síndrome de quemarse por el trabajo (burnout) (en adelante SQT) (Akar, 2018) que contribuye al deterioro de la calidad de vida laboral (Carlotto y Câmara, 2019).

En la actualidad el SQT está adquiriendo gran relevancia ya que puede predecir directamente o mediar en los efectos de predictores más globales, como por ejemplo disfunciones de rol, sobrecarga de trabajo, etc., a través de múltiples indicadores de bienestar y desempeño, como mala salud, estrés, depresión , baja satisfacción y actividades laborales reducidas (Sabagh et al., 2018). La Organización Mundial de la Salud (OMS) lo reconoció en el año 2019 como un problema de salud derivado única y exclusivamente de la actividad laboral. En el CIE-11, que entrará en vigor en 2022, estará incluido dentro de "Problemas asociados al empleo o desempleo" (QD85). La OMS considera que es un síndrome resultado del estrés crónico en el lugar de trabajo que no se ha manejado con éxito.

Las cifras del SQT reportadas fluctúan entre el 43\%-88\% en maestros de educación infantil, del $43 \%-80 \%$ en docentes de la primaria, del 36.6\%-80\% en los de la secundaria, y en el ámbito universitario, las cifras se mueven entre el 68\%-94.7\% (Fernández-Puig et al., 2015; Oramas et al., 2007). Aunque la prevalencia sea elevada, los docentes que experimentan niveles graves de SQT se reducen a un número que fluctúa entre $3 \%$ y un $13 \%$ de afectados. A nivel internacional, los valores se sitúan en el 15\% en Portugal (Figueiredo-Ferraz et al., 2009), 21.4\% en Uruguay (Ratto et al., 2015), 35.5\% en México (Unda, 2010), o en Brasil la prevalencia es de 7.5\% para el Perfil 1 de SQT y 18,3\% de Perfil 2 (Carlotto y Câmara, 2019).

El SQT es una respuesta al estrés laboral crónico. Es una experiencia subjetiva de carácter negativo compuesta por cogniciones, emociones y actitudes negativas hacia el trabajo, hacia las personas con las que se relaciona el individuo en su trabajo, en especial los clientes, y hacia el propio rol profesional. Como consecuencia de esta respuesta aparecen una serie de disfunciones conductuales, psicológicas y fisiológicas que pueden tener repercusiones nocivas para las personas y para la organización (Gil-Monte, 2011).

La definición más reconocida de SQT es de un síndrome cuyos síntomas son: baja realización personal en el trabajo, entendida como la tendencia de los profesionales a evaluar negativamente su actividad laboral y el ejercicio de la misma; agotamiento emocional, caracterizado por la experiencia de no poder dar más de sí mismos a nivel afectivo; y despersonalización, definida como el desarrollo de actitudes y sentimientos negativos hacia las personas destinatarias del trabajo (Maslach y Jackson, 1981). El cuestionario más utilizado internacionalmente para valorar el SQT es el Maslach Burnout Inventory (MBI) (Maslach y Jackson, 1986). Sus tres dimensiones se ajustan a la definición dada anteriormente (Maslach et al, 2001). Sin embargo, este instrumento presenta algunos problemas psicométricos (Olivares-Faúndez y Gil-Monte, 2009; Wheeler et al., 2011). Entre esos problemas se citan los siguientes: algunos estudios concluyeron que se deberían considerar solamente dos factores 
en lugar de los tres que propone el modelo, con los ítems de las escalas de Agotamiento emocional y Despersonalización formando un factor (lo que se ha llamado el «núcleo del burnout») y el segundo factor formado por los ítems de la escala de Realización personal en el trabajo (Holland, et al., 1994; Walkey y Green, 1992); la dimensión de Despersonalización presenta con demasiada frecuencia valores de consistencia interna demasiado bajos (Chao et al., 2011); y se debería evaluar de manera más amplia el SQT, pues no lo recoge en toda su amplitud. Otros autores opinan que la dimensión de Agotamiento emocional debería incorporar otros aspectos de agotamiento, como agotamiento cognitivo y físico, para recoger de una manera más completa la experiencia de agotamiento que experimentan los trabajadores (Halbesleben y Demerouti, 2005).

Una definición alternativa sobre el SQT fue elaborada por Gil-Monte (2005). Según este autor, el SQT es una respuesta psicológica al estrés laboral crónico de carácter interpersonal y emocional que aparece en los profesionales de las organizaciones de servicios que trabajan en contacto con los clientes o usuarios de la organización. Esta respuesta se caracteriza por cuatro síntomas: un deterioro cognitivo (pérdida de la ilusión por el trabajo o baja realización personal en el trabajo), un deterioro afectivo (agotamiento emocional y físico), aparición de actitudes y conductas negativas hacia los clientes y hacia la organización, en forma de comportamientos indiferentes, fríos, distantes y, en ocasiones, lesivos (indolencia) y, en algunos casos, estos síntomas se acompañan de sentimientos de culpa.

Para evaluar esos síntomas se desarrolló el «Cuestionario para la Evaluación del Síndrome de Quemarse por el Trabajo» (CESQT) ${ }^{1}$ (Gil-Monte, 2011; Gil-Monte y Olivares-Faúndez, 2011). Este cuestionario está basado en un modelo según el cual el deterioro cognitivo (bajas puntuaciones en Ilusión por el trabajo) y el deterioro afectivo (altas puntuaciones en Desgaste psíquico) son las primeras manifestaciones del SQT como respuesta al estrés laboral crónico. A estos síntomas le sigue la aparición de actitudes negativas hacia las personas con las que se trabaja (altas puntuaciones en Indolencia).

Además, algunos trabajadores, desarrollan altos sentimientos de culpa debido a estos síntomas, en especial por el trato desconsiderado e indolente hacia los clientes o usuarios de los servicios (Gil-Monte, 2005). Los sentimientos de culpa han sido identificados por diferentes autores como un síntoma del SQT (Carlotto y Câmara, 2019; Rabasa et al., 2016; Villaverde et al., 2019).

Desde que se iniciaron las investigaciones para explicar el proceso de desarrollo del SQT (Paine, 1982) hasta la actualidad (van Dam, 2016) son varios los estudios que vienen recomendado la necesidad de diferenciar entre tipos o grupos de individuos con SQT (Mahfoudh et al., 2019; Maltsev et al, 2019; Reyes-Oyola y Palomino-Devia, 2019; Villaverde et al., 2019) pues, aunque los profesionales que desarrollan esta patología comparten la mayoría de los síntomas, se observa que no en todos tiene las mismas consecuencias, o que su intensidad varía. Además, diferenciar perfiles en el desarrollo del SQT pueden ayudar a diferenciarlo de otras patologías con las que comparte síntomas, como la depresión (Havrylets et al., 2018; Lang et al., 2017; Rabasa et al., 2016; Yadollahi et al., 2016).

1 El nombre del CESQT en publicaciones en inglés es Spanish Burnout Inventory (SBI). 
Tomando como referencia el modelo teórico desde el que se ha construido el cuestionario, la evaluación con el cuestionario CESQT permite diferenciar dos perfiles de individuos en el desarrollo del SQT incorporando la variable «Sentimientos de culpa», ayudando a diferenciarlo de otros problemas de salud y sus consecuencias (Gil-Monte, 2012). El Perfil 1 conduce a la aparición de un conjunto de sentimientos y conductas vinculados al estrés laboral que originan una forma moderada de malestar, pero que no incapacita al individuo para el ejercicio de su trabajo, aunque podría realizarlo mejor. Este perfil se caracteriza por la presencia de baja ilusión por el trabajo, junto a altos niveles de desgaste psíquico e indolencia, pero los individuos no presentan niveles críticos de sentimientos de culpa, pues la indolencia funciona como estrategia de afrontamiento funcional para manejar el estrés laboral y sus síntomas. El Perfil 2 constituye con frecuencia un problema más serio que identificaría a los individuos más deteriorados por el desarrollo del SQT. Además de los síntomas anteriores, los individuos presentan niveles más altos de sentimientos de culpa.

El CESQT tiene algunas ventajas sobre otros instrumentos utilizados en la evaluación del SQT. Entre esas ventajas están: que es un instrumento basado en un modelo teórico con un desarrollo previo al modelo psicométrico y que sustenta a éste (Gil-Monte, 2005); aunque alguna de sus dimensiones son similares a las del MBI, el CESQT incorpora la evaluación de los sentimientos de culpa y dos perfiles de SQT, además incluye ítems para evaluar aspectos físicos del agotamiento y no solo aspectos psicológicos (Gil-Monte, 2011); y no presenta insuficiencias psicométricas cuando se aplica en idiomas diferentes al español (Bosle y Gil-Monte, 2010; Figueiredo-Ferraz et al., 2014; Gil-Monte et al., 2017; Misiolek et al., 2016; Vela, 2019; Viotti et al., 2015).

Los resultados obtenidos en estudios previos sobre las características psicométricas del CESQT han respaldado la estructura de cuatro factores mediante análisis factorial exploratorio y análisis factorial confirmatorio, y han obtenido resultados adecuados de consistencia interna, mediante el índice alfa de Cronbach, para las cuatro subescalas. Estos resultados se han obtenido en estudios realizados en España (Gil-Monte y Manzano-García, 2015), Italia (Viotti et al., 2015), México (Gil-Monte y Zúñiga-Caballero, 2010), Chile (Olivares y GilMonte, 2007), y Polonia (Misiolek et al., 2016).

También se ha obtenido valores adecuados para la validez concurrente entre el CESQT y el MBI. Los valores de correlación para las escalas de estos dos cuestionarios van de .35 a .52 para la correlación entre Ilusión por el trabajo y Realización personal en el trabajo, de .68 a .77 para la correlación entre Desgaste psíquico y Agotamiento emocional, y de .39 a .52 para la correlación entre Indolencia y Despersonalización (Gil-Monte y Olivares-Faúndez et al., 2011; Guidetti et al, 2018; Viotti et al., 2015), e incluso valores superiores en estudios menos recientes. Estos valores se pueden considerar moderados y aceptables (Cohen, 1988), lo que implica que el constructo teórico del SQT estimado mediante el CESQT es similar al de otras escalas aceptadas y utilizadas internacionalmente.

Dado que no existen estudios sobre la adaptación y validación del cuestionario al valenciano/catalán es por los que se realiza este trabajo, cuyo objetivo es analizar las propiedades psicométricas del cuestionario CESQT en valenciano/catalán que permiten evaluar el SQT 
en el contexto laboral de la educación, realizándose un análisis psicométrico de los ítems y análisis factorial confirmatorio.

\section{Método}

\section{Participantes}

La muestra estuvo formada por 1359 docentes no universitarios, de los cuales 435 eran hombres (32\%) y 891 mujeres $(65.56 \%)$ La media de edad de los participantes fue de 46.03 años $(\mathrm{DT}=9.18, \min .=20$ años, máx. $=67)$. La media de antigüedad en la profesión de los participantes en el estudio fue de 17.26 años $(\mathrm{DT}=10.21, \min =0$ años, Max. = 45). El $71.3 \%$ de la muestra era funcionario de carrera, el $60 \%$ trabajaba en un Instituto de Educación Secundaria y el $23.6 \%$ en un Centro de Educación Infantil y Primaria.

\section{Instrumento}

A los participantes se les ofreció la posibilidad de elegir responder al cuestionario en papel en valenciano/catalán o castellano del «Cuestionario para la Evaluación del Síndrome de Quemarse por el Trabajo» (CESQT) (Gil-Monte, 2011). Este instrumento fue traducido al valenciano/catalán por el Servicio de Traducción y Asesoramiento del Valenciano, que tiene asignada la competencia de asesorar en cuestiones lingüísticas a la Generalitat siguiendo la normativa lingüística de la Acadèmia Valenciana de la Llengua, a petición del Instituto Valenciano de Seguridad e Higiene en el Trabajo (INVASSAT). Este instrumento se compone de 20 ítems distribuidos en cuatro dimensiones denominadas: (a) Ilusión por el trabajo: deseo del individuo de alcanzar las metas laborales, ya que suponen una fuente de placer personal, 5 ítems, (p.e., "El meu treball em suposa un repte estimulant"), (b) Desgaste psíquico: agotamiento emocional y físico debido a que, en el trabajo, se tiene que tratar a diario con personas que presentan o causan problemas, 4 ítems, (p.e., "Em sent desgastat emocionalment"), (c) Indolencia: actitudes negativas de indiferencia y cinismo hacia los «clientes de la organización» 6 ítems, (p.e., "Pense que tracte amb indiferència alguns alumnes"), y (d) Culpa: sentimientos de culpa por las actitudes negativas desarrolladas en el trabajo, en especial con las personas hacia las que se trabaja, 5 ítems, (p.e., "Em sent culpable per alguna de les meues actituds en el trebal"). Los ítems se responden mediante una escala de frecuencia de cinco grados (0 "Mai" a 4 "Molt sovint: Tots els dies"), siendo bajas las puntuaciones más próximas a 0 y altas las más próximas a 4.

\section{Procedimiento}

La muestra estuvo formada por personal trabajador público del sector docente no universitario de la provincia de Valencia que trabajaban en centros donde se realizó la evaluación de riesgos psicosociales entre los años 2018 y 2019. Para su realización, previamente se comunicó por el personal investigador al director del centro, y a los representantes legales de los trabajadores su realización, llegando a un acuerdo con ellos sobre la fecha de su realización, y el procedimiento que se iba a seguir. La entrega y recogida de los cuestionarios siguió el proceso característico del procedimiento seguido por la Unidad de Investigación Psicosocial de la Conducta Organizacional (UNIPSICO). Al personal trabajador se le ofreció la posibilidad de elegir entre responder al cuestionario en valenciano/catalán o castellano. La 
distribución de los cuestionarios la realizó un miembro del equipo de investigación quien, tras la explicación del objetivo del estudio, y que no debían dejarse respuestas en blanco, se les garantizó la confidencialidad de las respuestas, lo entregaba en mano al personal trabajador para ser recogido por el mismo investigador cuando se terminó de cumplimentar. La participación de todos los participantes fue voluntaria. Antes de iniciar la recogida de datos se comprobó que el procedimiento seguía los principios éticos del INVASSAT (Generalitat Valenciana). El estudio aplicó los principios de la Declaración de Helsinki (World Medical Association, 2013), teniendo especial cuidado en que los cuestionarios respondidos fueran anónimos, cuidando la confidencialidad de los datos al tener solamente acceso el personal investigador que intervino en el proceso y la no discriminación de los participantes, dado que la participación de toda la plantilla del centro educativo fue voluntaria.

\section{Análisis de datos}

Se realizó un análisis de los ítems considerando los estadísticos descriptivos para valorar si ajustaban a una distribución normal. Se calculó el valor de la media, desviación típica, asimetría y curtosis. Otros criterios para valorar la calidad de un ítem fue el cálculo de la relación entre el ítem y el total de la escala de la que forma parte, pues de esta manera se puede valorar el grado en que un ítem contribuye a evaluar el constructo para el que ha sido diseñado. Los criterios para evaluar este aspecto de los ítems fueron: (1) correlación corregida de cada ítem con el total de la escala (homogeneidad corregida) y (2) fiabilidad de la escala al eliminar el ítem.

Para la validez de constructo se utilizó el análisis factorial confirmatorio (AFC). El método de estimación fue máxima verosimilitud (maximum likelihood, ML), dado que los valores de asimetría no excedían el valor \pm 2 , pudiéndose afirmar que la distribución es normal (Miles y Shevlin, 2005, p.74), y la escala de respuesta tiene un número de categorías de respuesta igual a 5 (Lloret-Segura, et al., 2014). Para valorar el ajuste global del modelo, además del índice chi cuadrado $(\chi 2)$ se consideraron los siguientes índices que no están afectados por el tamaño de la muestra. El Adjusted Goodness of Fit Index (AGFI) que mide la cantidad relativa de varianza explicada por el modelo. El Non-Normed Fit Index (NNFI) y el Comparative Fit Index (CFI) que son indicadores del ajuste relativo del modelo. Para estos índices los valores superiores a .90 se consideran indicadores de un ajuste aceptable del modelo (Bentler, 1992; Hoyle, 1995). El Root Mean Square Error of Aproximation (RMSEA) estima la cantidad global de error existente en el modelo. Los valores entre .05 y .08 indican un ajuste adecuado del modelo (Beaubien, 1999; Hair et al, 1995).

Para el análisis de fiabilidad se calculó el valor del alfa de Cronbach. También se realizó un análisis descriptivo de las escalas (media, desviación típica, asimetría y curtosis), y de correlación de Pearson. El análisis de ítems, y los análisis de fiabilidad de las escalas se realizó con el programa estadístico SPSS, versión 26. Los análisis para probar la validez de constructo se realizaron con el programa AMOS, versión 26.

\section{Resultados}

Para alcanzar el objetivo del estudio se realizaron tres tipos de análisis de datos: (1) análisis descriptivo de los ítems, (2) evaluación de la estructura factorial del CESQT, y (3) análisis de la consistencia interna de las escalas. 


\section{Análisis descriptivo de los ítems}

Se observa que las medias más elevadas se obtuvieron para los ítems que forman la dimensión Ilusión por el trabajo, que se caracterizan porque altas puntuaciones indican bajos niveles del SQT. El ítem con el valor más alto para la media fue el ítem 10 (Pense que el meu treball m'aporta coses positives) $(\mathrm{M}=3.35)$, que pertenece a la escala de Ilusión por el trabajo. Por el contrario, las medias más bajas corresponden a los ítems de las escalas de

Tabla 1.- Estadísticos descriptivos para los items de la escalas de Cuestionario para la Evaluación del Sindrome de Quemarse por el trabajo CESQT.

\begin{tabular}{|c|c|c|c|c|c|}
\hline $\mathrm{N}^{0}$ Item & Media (DT) & Homogeneidad corregida & Asimetría & Curtosis & Alfa sin ítem \\
\hline \multicolumn{6}{|c|}{ Ilusión por el trabajo, $\alpha=.87_{(86-88)}$} \\
\hline 1 & $3.24(0.80)$ & .67 & -0.98 & 0.85 & .86 \\
\hline 5 & $3.26(0.91)$ & .71 & -1.29 & 1.43 & .85 \\
\hline 10 & $3.35(0.79)$ & .70 & -1.24 & 1.58 & .85 \\
\hline 15 & $3.29(0.83)$ & .76 & -1.20 & 1.29 & .83 \\
\hline 19 & $3.11(0.92)$ & .69 & -1.01 & 0.81 & .85 \\
\hline \multicolumn{6}{|c|}{ Desgaste psíquico, $\alpha=.81_{(799.83)}$} \\
\hline 8 & $1.76(1.12($ & .65 & 0.13 & 0.65 & .75 \\
\hline 12 & $1.60(1.06)$ & .63 & 0.28 & 0.47 & .76 \\
\hline 17 & $1.79(1.10)$ & .62 & 0.16 & 0.62 & .77 \\
\hline 18 & $1.58(1.10)$ & .61 & 0.39 & 0.54 & .77 \\
\hline \multicolumn{6}{|c|}{ Indolencia, $\alpha=.69_{(.67 .72)}$} \\
\hline 2 & $1.05(1.02)$ & .51 & 0.84 & 0.13 & .62 \\
\hline 3 & $1.20(0.98)$ & .51 & 0.77 & 0.31 & .62 \\
\hline 6 & $0.91(0.86)$ & .37 & 0.88 & 0.78 & .67 \\
\hline 7 & $0.48(0.80)$ & .40 & 2.09 & 5.01 & .66 \\
\hline 11 & $1.41(1.09)$ & .37 & 0.41 & 0.57 & .67 \\
\hline 14 & $1.01(0.95)$ & .39 & 0.76 & 0.15 & .66 \\
\hline \multicolumn{6}{|c|}{ Culpa, $\alpha=.79_{(.77-81)}$} \\
\hline 4 & $0.98(1.03)$ & .50 & 1.26 & 1.32 & .79 \\
\hline 9 & $0.91(0.84)$ & .58 & 0.95 & 1.12 & .75 \\
\hline 13 & $0.65(0.73)$ & .64 & 1.26 & 2.43 & .73 \\
\hline 16 & $0.83(0.76)$ & .59 & 1.07 & 2.18 & .75 \\
\hline 20 & $0.77(0.81)$ & .59 & -0.98 & 0.85 & .74 \\
\hline
\end{tabular}

Nota 1. El número del ítem corresponde a su posición en el cuestionario.

Nota 2. Los valores alfa de Cronbach se ofrecen con intervalos de confianza 95\%.

$\mathrm{DT}=$ desviación típica 
Indolencia y Culpa. Con respecto al ítem con el valor más bajo, fue el ítem 7 (Pense que tracte amb indiferència alguns alumnes $)(\mathrm{M}=0.48)$ que pertenece a la escala de Indolencia. Para todos los ítems se obtuvieron valores de asimetría entre \pm 2 . De los 20 ítems del cuestionario solamente un ítem excedió ligeramente el rango de \pm 2 para el valor de la asimetría, este fue el ítem 7 (As = 2.09) que pertenece a la dimensión de Indolencia. Para curtosis, tres ítems superaros el valor de \pm 2 . Estos ítems fueron el $7(\mathrm{Cu}=5.01), 13$ (Tinc remordiments per alguns dels meus comportaments en el treball) $(\mathrm{Cu}=2.43)$, y 16 (Pense que hauria de

Tabla 2.- Prueba t para los items de la escalas de Cuestionario para la Evaluación del Sindrome de Quemarse por el trabajo CESQT

\begin{tabular}{|c|c|c|c|}
\hline $\mathrm{N}^{0}$ Ítem & Media Hombres & Media mujeres & $\mathrm{t}$ \\
\hline \multicolumn{4}{|c|}{ Ilusión por el trabajo } \\
\hline 1 & 3.13 & 3.31 & $-3.79 * * *$ \\
\hline 5 & 3.07 & 3.36 & $-5.24 * * *$ \\
\hline 10 & 3.23 & 3.42 & $-4.06 * * *$ \\
\hline 15 & 3.20 & 3.35 & $-3.16^{* *}$ \\
\hline 19 & 2.96 & 3.18 & $-4.00 * * *$ \\
\hline \multicolumn{4}{|c|}{ Desgaste psíquico } \\
\hline 8 & 1.67 & 1.81 & $-2.07 *$ \\
\hline 12 & 1.47 & 1.67 & $-3.35 * *$ \\
\hline 17 & 1.57 & 1.94 & $-5.18 * * *$ \\
\hline 18 & 1.47 & 1.65 & $-2.80 * *$ \\
\hline \multicolumn{4}{|c|}{ Indolencia } \\
\hline 2 & 1.08 & 1.02 & 0.98 \\
\hline 3 & 1.28 & 1.15 & $2.20^{*}$ \\
\hline 6 & 0.84 & 0.94 & $1.98^{*}$ \\
\hline 7 & 0.59 & 0.42 & $3.80^{* * *}$ \\
\hline 11 & 1.63 & 1.30 & $5.13^{* * *}$ \\
\hline 14 & 1.20 & 0.92 & $5.11 * * *$ \\
\hline \multicolumn{4}{|c|}{ Culpa } \\
\hline 4 & 1.14 & 0.91 & $3.83^{* * *}$ \\
\hline 9 & 0.92 & 0.91 & 0.10 \\
\hline 13 & 0.69 & 0.63 & 1.30 \\
\hline 16 & 0.91 & 0.79 & $2.63^{* *}$ \\
\hline 20 & 0.81 & 0.75 & 1.08 \\
\hline
\end{tabular}


demanar disculpes a algú pel meu comportament $)(\mathrm{Cu}=2.18)$. El primero pertenece a la dimensión de Indolencia y los otros dos a Culpa.

Como el género es una variable sociodemográfica que podría establecer diferencias significativas en los niveles de burnout (Gil-Monte, y Olivares Faúndez, 2011), se realizaron análisis de diferencias de medias para el sexo para todos los ítems (Tabla 2). Se obtuvieron diferencias significativas entre hombres y mujeres en todos los ítems excepto en el ítem 2 (No m'abellix atendre alguns alumnes), que pertenece a la escala de Indolencia, el ítem 9 (Em sent culpable per alguna de les meues actituds en el treball), el ítem 13 y el ítem 20 (Em sent malament per algunes coses que he dit en el treball), todos ellos de la escala de Culpa. Los hombres obtuvieron medias más elevadas que las mujeres con diferencias estadísticamente significativas para todos los ítems correspondientes a la variable de Indolencia, excepto el ítem 6, y para los de la variable Culpa. Por el contrario, las mujeres obtuvieron medias más elevadas con diferencia estadísticamente significativa con los hombres para todos los ítems de Ilusión por el trabajo y de Desgaste psíquico, y para el ítem 6 de Indolencia.

\section{Análisis de la estructura factorial}

Se realizó un análisis factorial confirmatorio (AFC) con el fin de probar el ajuste de un modelo hipotetizado de cuatro factores (Ilusión por el trabajo, Desgate psíquico, Indolencia, y Culpa). El modelo hipotetizado presentó un ajuste adecuado con todos los índices de ajuste, excepto con el índice $\chi 2(164)(800.71, \mathrm{p}<.001)$ que está afectado por el tamaño de la muestra. La cantidad relativa de varianza explicada por el modelo fue adecuada, alcanzando el AGFI un valor superior a $.90(\mathrm{AGFI}=.926)$. El ajuste del modelo resultó también adecuado al considerar la cantidad global de error existente en el modelo, RMSEA $=.053_{(.050-057)}$. También fueron adecuados los valores obtenidos para los índices con los que se evaluó el ajuste relativo del modelo, pues tanto el NNFI como el CFI presentaron valores superiores a $.90 ; \mathrm{NNFI}=.919$ y $\mathrm{CFI}=.930$.

Todas las saturaciones factoriales resultaron estadísticamente significativas. El valor más bajo se obtuvo para la saturación del ítem 11 en el factor Indolencia $(\lambda=.44)$. Para Ilusión por el trabajo, el ítem que obtuvo una mayor saturación fue el $15(\lambda=.83)$, mientras que el que obtuvo una saturación menor fue el $1(\lambda=.71)$. Para Desgaste psíquico, el ítem que obtuvo una saturación mayor fue el $8(\lambda=.74)$ y la saturación menor fue para el $17(\lambda=$ .70). Para Indolencia, el ítem que obtuvo una saturación mayor fue el $3(\lambda=.64)$, mientras el menor fue el $11(\lambda=.44)$, y para Culpa el ítem que obtuvo una saturación mayor en el factor fue el $13(\lambda=.77)$, mientras que el menor fue el $4(\lambda=.55)$.

Todas las relaciones entre las dimensiones del CESQT resultaron estadísticamente significativas. Como era de esperar según las definiciones de las dimensiones del CESQT, las relaciones entre Ilusión por el trabajo y el resto de las escalas resultaron negativas, mientras que las relaciones entre el resto de las escalas fueron positivas. El resultado del análisis de covarianza estandarizada se encuentra en la Figura 1 obteniéndose una relación negativa entre la escala de Ilusión por el trabajo y Culpa (-.07), Indolencia (-.17), y Desgaste (-.15). El resto de las relaciones entre las escalas que forman parte de CESQT fueron positivas, siendo todas ellas estadísticamente significativas $\mathrm{p}<.001$. 
Figura 1.- Resultados del modelo factorial hipotetizado para el Cuestionario para la

Evaluación del Síndrome de Quemarse por el trabajo (CESQT)

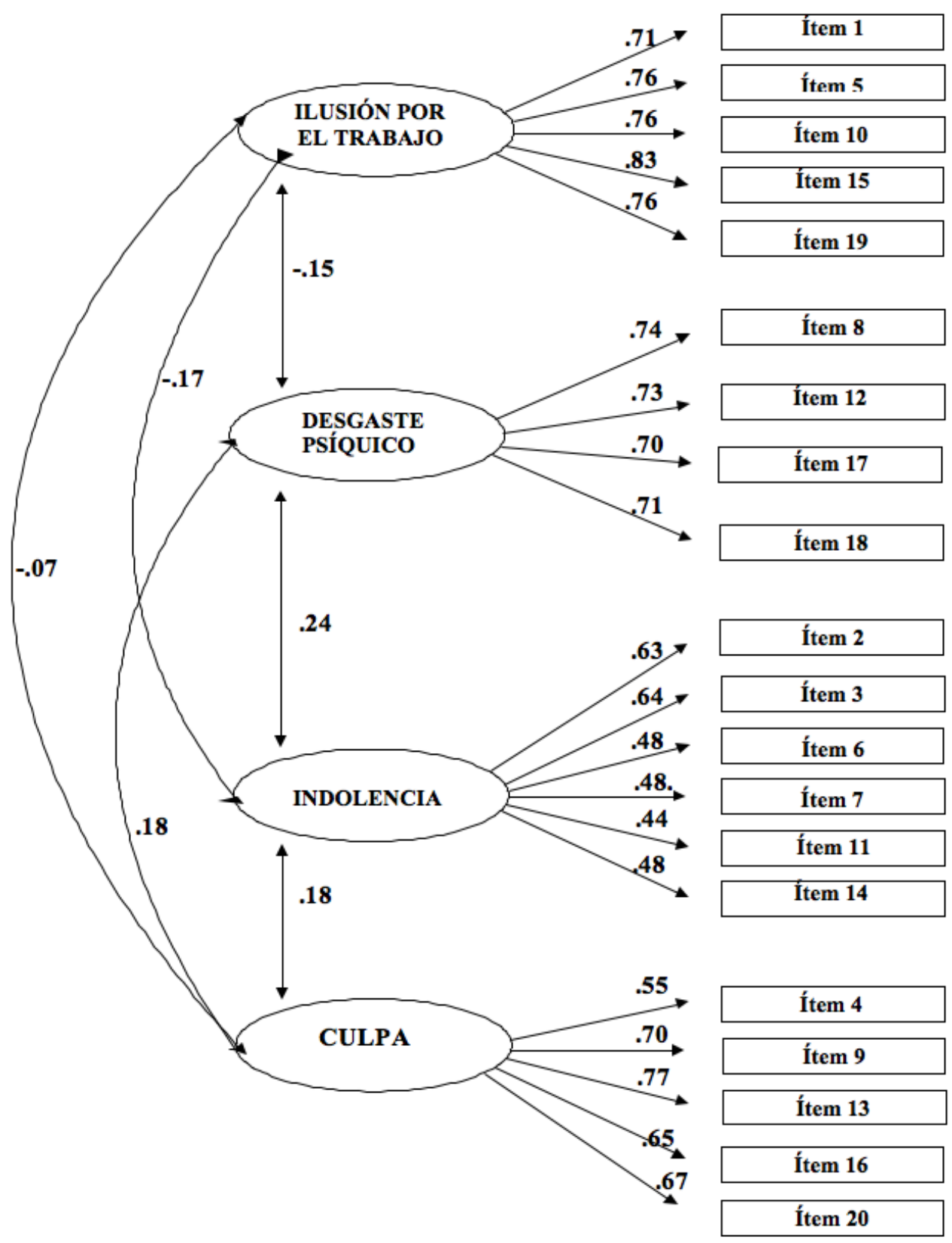




\section{Análisis de consistencia interna}

Se evaluó la consistencia interna para las escalas del instrumento mediante el coeficiente alfa de Cronbach. El coeficiente alfa para el conjunto de los 15 ítems que forman las escalas de Ilusión por el trabajo (invertida), Desgaste psíquico e Indolencia fue $\alpha=.83_{(.81-84)}$. Todas las escalas presentaron valores alfa de Cronbach superior a .70 , excepto la de Indolencia, $\alpha=.69_{(.67-.72)}$ (Tabla 1). En todas las escalas todos los ítems contribuyeron a incrementar la consistencia interna de la escala de la que forman parte, pues su eliminación disminuía el valor del alfa de Cronbach en todos los casos, con excepción del ítem 4 que el eliminarlo no se modificaba el valor del alfa de Cronbach para la escala de Culpa (Tabla 1).

\section{Análisis de escalas}

La Tabla 3 muestra los estadísticos descriptivos para las subescalas del CESQT. Las escalas se ajustaron a la distribución normal, pues los valores de asimetría oscilaron entre -1 y +1, con excepción de la escala de Ilusión por el trabajo que se desvió ligeramente (As = -1.06). Todas las correlaciones entre las escalas del CESQT fueron significativas. Según la descripción de las dimensiones del CESQT, las correlaciones entre Ilusión por el trabajo y el resto de escalas fueron negativas, mientras que las relaciones entre el resto de escalas fueron positivas. La correlación más alta se encontró entre las escalas de Indolencia y Culpa (.40, $\mathrm{p}<.001)$, y la correlación más débil entre las escalas de Ilusión por el trabajo y Culpa (-.16, $\mathrm{p}<.001)$ (Tabla 3).

Tabla 3.- Estadísticos descriptivos y correlaciones para las variables de la escalas de Cuestionario para la Evaluación del Síndrome de Quemarse por el trabajo (CESQT)

\begin{tabular}{lcccccccc}
\hline & Media (DT) & As & Cu & Rango & 1 & 2 & 3 & 4 \\
\hline 1 Ilusión por el trabajo & $3.25(0.69)$ & -1.06 & 1.15 & $0-4$ & 1 & & & \\
2 Desgaste psíquico & $1.68(0.88)$ & 0.32 & -0.23 & $0-4$ & $-.27 * * *$ & 1 & & \\
3 Indolencia & $1.01(0.60)$ & 0.71 & 0.78 & $0-4$ & $-.35^{* * * *}$ & $.34 * * *$ & 1 & \\
4 Culpa & $0.83(0.62)$ & 0.90 & 1.55 & $0-4$ & $-.16^{* * *}$ & $.29^{* * * *}$ & $.40^{* * *}$ & 1 \\
\hline
\end{tabular}

$* * * \mathrm{p}<.001$

$\mathrm{DT}=$ Desviación típica, $\mathrm{As}=$ Asimetría, $\mathrm{Cu}=$ Curtosis.

Nota 1. Los valores alfa de Cronbach se ofrecen con intervalos de confianza 95\%.

\section{Discusión}

El objetivo de este estudio fue analizar las propiedades psicométricas de la traducción al valenciano/catalán del CESQT en una muestra de personal docente público de la provincia de Valencia, con el fin de concluir sobre la validez y fiabilidad del cuestionario en un idioma diferente al que fue creado. Para alcanzar el objetivo del estudio se ha probado la calidad psicométrica de los ítems que forman parte del cuestionario, su estructura factorial y la consistencia interna de las cuatro escalas que lo forman. 
Los resultados del estudio indican que las propiedades psicométricas del cuestionario son adecuadas en la muestra del estudio, y han confirmado la estructura factorial hipotetizada al ajustar al modelo de medida de cuatro factores, formado por los constructos Ilusión por el trabajo, Desgaste psíquico, Indolencia y Culpa. El ajuste del modelo fue bueno según el GFI y aceptable según los índices de incremento NNFI y CFI que presentaron valores superiores al umbral recomendado para aceptar el ajuste de un modelo de AFC (Bentler, 1992; Hoyle, 1995). También fue bueno el ajuste del modelo al considerar los residuales, pues el valor del índice RMSEA se encontró entre .05 y .08 (Browne y Cudeck, 1993; Hair et al., 1995). Estos resultados son similares a los obtenidos con muestras de personal médico mexicano (Gil-Monte y Zúñiga y Caballero, 2010) o de maestros (Gil-Monte et al., 2009), o de otros países de distintos sectores (Bosle y Gil-Monte, 2010; Figueiredo-Ferraz et al., 2014; GilMonte et al., 2017; Misiolek et al., 2016; Vela, 2019; Viotti et al., 2015), y permiten afirmar que el modelo de medida del CESQT presenta una estructura que hace que pueda ser utilizado en diferentes contextos profesionales y geográficos.

Los resultados obtenidos en el análisis de asimetría de los ítems siguen el patrón de estudios previos realizados en profesionales de la salud con la versión española del cuestionario (Gil-Monte y Manzano García, 2015; Gil-Monte y Zúñiga Caballero, 2010), de igual manera para la curtosis en profesionales de la salud italianos (Gil-Monte et al., 2017). Para todos los ítems los valores de asimetría y curtosis estuvieron dentro del intervalo que se considera aceptable para concluir que la distribución de las puntuaciones se ajusta a la distribución normal (Miles y Gilbert, 2005). Cuando se consideró el valor de la media de los ítems se observó que el valor más alto y más bajo de la media de los ítems en las escalas de Ilusión por el trabajo son el ítem 10 y 19, respectivamente, Desgaste psíquico ítems 17 y 18, respectivamente, e Indolencia ítems 11 y 6, respectivamente, y en la escala de Culpa la media más elevada se encontró en el ítem 4, la más baja en el ítem 13. Estos resultados son similares a los obtenidos en otros estudios (Gil-Monte et al., 2015; Guidetti et al., 2018) y también, en el caso de los ítems con puntuaciones más bajas (Gil-Monte y Zúñiga Caballero, 2010).

Considerando la estructura factorial del CESQT los resultados confirmaron la hipótesis formulada, pues el modelo de cuatro factores obtuvo un ajuste adecuado a los datos. Estos resultados permiten concluir que el modelo factorial del CESQT en su versión en valenciano/ catalana se ajusta de manera adecuada al modelo teórico original (Gil-Monte, 2011), que incluye cuatro dimensiones o síntomas de SQT: Ilusión por el trabajo, Desgaste psíquico, Indolencia y Culpa.

La consistencia interna de las cuatro dimensiones fue satisfactoria. Los valores alfa de Cronbach van desde el $\alpha=.69_{(.67-72)}$ en Indolencia a $\alpha=.87_{(.86-88)}$ en Ilusión por el trabajo. Aunque para la escala de Indolencia el valor obtenido fue ligeramente inferior al umbral recomendado por Nunnally (1978) $(\alpha=.70)$, no se puede afirmar que ese valor sea pobre o inaceptable (George y Mallery, 2003, p. 231); no suponiendo esto una amenaza para la fiabilidad del instrumento dado el número de ítems que componen la escala. Este resultado está en la línea de los estudios previos realizados con maestros (Gil-Monte et al., 2009; GilMonte et al., 2015), en los que se obtuvieron valores alfa de Cronbach superiores a .70 para las cuatro dimensiones del CESQT; por lo tanto, confiere validez interna a las dimensiones 
del CESQT para su aplicación a aquellas personas de habla valenciana o catalana, y concretamente, en el colectivo docente.

Los valores de las correlaciones muestran una relación negativa entre Ilusión por el trabajo y el resto de escalas, siendo positivas las relaciones entre el resto de las escalas. Estos resultados eran los esperados en nuestra hipótesis de partida puesto que la Ilusión por el trabajo se comportaría como un factor protector de Desgaste psíquico, Indolencia y Culpa, mientras que el resto de factores estarán interrelacionados de forma positiva entre ellos (Gil-Monte, 2005; Gil-Monte y Olivares-Faúndez, 2011).

Los resultados del estudio permiten concluir que el cuestionario CESQT en valenciano/ catalán tiene propiedades psicométricas adecuadas para el estudio del SQT en profesionales del sector docente de la provincia de Valencia.

La relevancia del estudio es que contribuye a probar la validez psicométrica del cuestionario CESQT para personas de habla valenciana o catalana, permitiendo su uso para profesionales de la docencia en la Comunidad Valenciana, Islas Baleares y Cataluña que podrán responder el cuestionario en su lengua materna, sumándose a otros estudios que ya han probado esas características psicométricas en idiomas diferentes al castellano. Así, pues se consolida la validez transcultural del CESQT.

Como limitación de este estudio se encuentra que la muestra estuvo compuesta en su mayor parte por mujeres, pudiendo esto haber afectado a los resultados. Por ello, es recomendable que en futuros estudios se desarrollen investigaciones comparativas considerando algunas variables de corte sociodemográfico que influyen significativamente en el SQT, como el género, tipo de trabajo u otras. Por otra parte, Moreno-Jiménez, et al. (2003) consideran que el SQT no debería plantearse exclusivamente en términos de procesos intrapsíquicos, sino de prácticas sociales (culturales, económicos y políticos), de manera que los resultados tengan un sesgo sociopolítico fruto de esa situación.

Futuros estudios deben explorar la evidencia de puntos de corte con carácter de diagnóstico clínico, esto es, a partir de qué valor o de qué percentil los sujetos presentan niveles de SQT con una frecuencia tal que deteriora su salud con el fin de analizar el impacto epidemiológico real del SQT como enfermedad laboral.

\section{Referencias}

Akar, H. (2018). The Relationships between quality of work life, school alienation, burnout, affective commitment and organizational citizenship: A study on teachers. European Journal of Educational Research, 7(2), 169-180.

Beaubien, J. M. (1999). Structural Equation Modeling with LISREL, PRELIS, and SIMPLIS: Basic concepts, applications, and programming. Personnel Psychology, 52(3), 827-830.

Bentler, P. M. (1992). On the fit of models to covariances and methodology to the Bulletin. Psychological Bulletin, 112(3), 400. doi: 10.1037/0033-2909.112.3.400

Bosle, A., y Gil-Monte, P. R. (2010). Psychometric Properties of the Spanish Burnout Inventory in German Professionals: Preliminary Results. Ansiedad y Estrés, 16(2/3), 283-291. 
Browne, M. W., y Cudeck, R. (1993). Alternative ways of assessing model fit. En K. A. Bollen y L. S. Long (Eds.), Testing Structural Equation Models (pp.136-162). Newbury Park, CA: Sage

Carlotto, M. S., y Câmara, S. G. (2019). Prevalence and predictors of Burnout Syndrome among public elementary school teachers. Análise Psicológica, 37(2), 135-146. doi: 10.14417/ap.1471

Chao, S. F., McCallion, P., y Nickle, T. (2011). Factorial validity and consistency of the Maslach Burnout Inventory among staff working with persons with intellectual disability and dementia. Journal of Intellectual Disability Research, 55, 529-536. doi: 10.1111/j.1365-2788.2011.01413.x

Cohen, J. (1977). Statistical power analysis for the behavioral sciences (Rev. ed. ed.) Lawrence Erlbaum Associates, Inc, Hillsdale, NJ.

Fernández-Puig, V., Longás, J., Chamarro, A., y Virgili, C. (2015). Evaluando la salud laboral de los docentes de centros concertados: el Cuestionario de Salud Docente. Journal of Work and Organizational Psychology, 30, 1-11. doi: 10.1016/j.rpto.2015.07.001

Figueiredo-Ferraz, H., Gil-Monte, P. R., y Grau-Alberola, E. (2009). Prevalencia del Síndrome de Quemarse por el Trabajo (burnout) en una muestra de maestros portugueses. Aletheia, 29, 6-15. doi: 10.11144/Javeriana.upsy9-1.vfce.

Figueiredo-Ferraz, H., Gil-Monte, P. R., Queirós, C., y Passos, F. (2014). Validação factorial do «Spanish Burnout Inventory» em polícias portugueses. Psicologia: Reflexao e Crítica, 27, 291-299. doi: 10.1590/1678-7153.201427209.

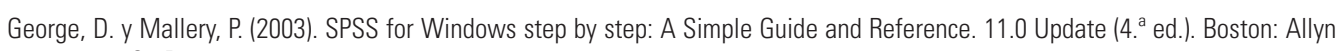
\&y Bacon

Gil-Monte, P. R. (2005). El síndrome de quemarse por el trabajo (burnout). Una enfermedad laboral en la sociedad del bienestar. Madrid: Pirámide.

Gil-Monte, P. R. (2011). CESQT. Cuestionario para la Evaluación del Síndrome de Quemarse por el Trabajo. Madrid: TEA Ediciones.

Gil-Monte, P. R. (2012). Influence of guilt on the relationship between burnout and depression. European Psychologist, 17, 231-236. doi: 10.1027/1016-9040/a000096

Gil-Monte, P. R. y Manzano-García, G. (2015). Psychometric properties of the Spanish Burnout Inventory among staff nurses. Journal of Psychiatric and Mental Health Nursing, 22, 756-763. doi: 10.1111/jpm.12255

Gil-Monte, P. R. y Olivares-Faúndez, V. (2011). Psychometric properties of the «Spanish Burnout Inventory» in Chilean professionals working to physical disabled people. The Spanish Journal of Psychology, 14, 441-451. doi: 10.5209/rev_SJOP. 2011.v14. $\mathrm{n} 1.40$

Gil-Monte, P. R., Unda S., y Sandoval J. I. (2009). Validez factorial del “Cuestionario para la Evaluación del Síndrome de Quemarse por el Trabajo" (CESQT) en una muestra de maestros mexicanos. Salud Mental, 32, 205-214.

Gil-Monte, P. R., Viotti, S., y Converso, D. (2017). Propiedades psicométricas del «Cuestionario para la Evaluación del Síndrome de Quemarse por el Trabajo»(CESOT) en profesionales de la salud italianos: una perspectiva de género. Liberabit, 23(2), 153-168.

Guidetti, G., Viotti, S., Gil-Monte, P. R., y Converso, D. (2018). Feeling guilty or not guilty. Identifying burnout profiles among Italian Teachers. Current Psychology, 37, 769-780. doi: 10.1007/s12144-016-9556-6.

Gil-Monte, P. R. y Zúñiga-Caballero, L. C. (2010). Validez factorial del «Cuestionario para la Evaluación del Síndrome de Quemarse por el Trabajo» (CESQT) en una muestra de médicos mexicanos. Universitas Psychologica, 9, 169-178.

Hair, J. H., Anderson, R. E., Tatham, R. L., \&y Black, W. C. (1995) Multivariate data analysis. (5th ed.). Prentice-Hall International.

Halbesleben, J. R. y Demerouti, E. (2005). The construct validity of an alternative measure of burnout investigating the English translation of the Oldenburg Burnout Inventory. Work and Stress, 19, 208-220. doi: 10.1080/02678370500340728

Havrylets, Y., Tukaiev, S., Rizun, V., y Khylko, M. (2018). State anxiety, mood, and emotional effects of negative TV news depend on burnout. doi.org/10.31234/osf.io/m3xv2

Holland, P. J., Michael, W. B., y Kim, S. (1994). Construct validity of the Educators Survey for a sample of middle school teachers. Educational and Psychological Measurement, 54, 822-829. doi:10.1177/001316449405 4003029

Hoyle, R. H. (1995). The Structural Equation Modeling approach: Basic concepts and fundamental issues. En: Hoyle, R. H. (ed). Structural equation modeling: Concepts, issues, and applications. Thousand Oaks, CA: Sage, p.1-15.

Lang, C. N., Steinfurt, J., y Odening, K. E. (2017). Avoiding sports-related sudden cardiac death in children with congenital channelopathy. Herz, 42, 162-170. doi:10.1007/s00059-017-4549-2. 
Lloret-Segura, S., Ferreres-Traver, A., Hernández-Baeza, A. y Tomás-Marco, I. (2014). El análisis factorial exploratorio de los ítems: una guía práctica, revisada y actualizada. Anales de Psicología, 30, 1151-1169. doi: 10.6018/analesps.30.3.199361

Mahfoudh, A., Bakhrouf, A., Rassas, I., Ammar, H., Bouzgarrou, L., Omrane, A., Henchi, A., Akrout, M., \&y Khalfallah, T. (2019). Influence of socioprofessional determinants on teachers' mental well-being. Giornale Italiano di Medicina del Lavoro ed Ergonomia. 41, 236-241.

Maltsev, V. P., Belousova, N. A., y Titarenko, N. N. (2019). Features of modern secondary school teacher's value-based attitude to health and emotional burnout. Revista Espacios, 40, 1-11.

Maslach, C. y Jackson, S. E. (1981). Maslach Burnout Inventory. Manual. Palo Alto, CA: Consulting Psychologists Press.

Maslach, C., y Jackson, S. E. (1986). Maslach Burnout Inventory. Manual. Palo Alto, CA: Consulting Psychologists Press.

Maslach, C., Schaufeli, W. B., y Leiter, M. P. (2001). Job burnout. Annual Review of Psychology, 52, 397-422. doi: 10.1146/annurev. psych.52.1.397

Miles, J., \& Shevlin, M. (2005). Applying regression \& correlation. A guide for students and researchers. London: Sage.

Misiolek, A., Gil-Monte, P. R., y Misiolek, H. (2016). Prevalence of burnout in Polish anesthesiologists and anesthetist nursing professionals: A comparative nonrandomized cross-sectional study. Journal of Health Psychology, 22, 465-474. doi: $10.1177 / 1359105315604377$

Moreno-Jiménez, B., Garrosa, E., Benevides-Pereira, A. M., y Gálvez, M. (2003). Estudios transculturales del burnout: Los estudios transculturales Brasil-España. Revista Colombiana de Psicología, 12, 9-18.

Nunnally, N. C. (1978). Psychometric theory. New York. McGraw-Hill.

Olivares, V. E. y Gil-Monte, P. R. (2007). Análisis de las propiedades psicométricas del «Cuestionario para la Evaluación del Síndrome de Quemarse por el Trabajo» (CESOT) en profesionales chilenos. Ansiedad y Estrés, 13, 229-240.

Olivares-Faúndez, V. y Gil-Monte, P. R. (2009). Análisis de las principales fortalezas y debilidades del «Maslach Burnout Inventory» (MBI). Ciencia \& Trabajo, 11, 160-167.

Oramas, A., Almirall, P., y Fernández, I. (2007). Estrés laboral y el síndrome de burnout en docentes venezolanos. Salud de los Trabajadores, 15, 71-88.

Paine, W. S. (1982). The burnout syndrome in context. En J. W. Jones (Ed.), The burnout syndrome: Current research, theory, interventions (pp. 1-29). Park Ridge: London House Press.

Rabasa, B., Figueiredo-Ferraz, H., Gil-Monte, P. R., y Llorca-Pellicer, M. (2016). The role of guilt in the relationship between teacher's job burnout syndrome and the inclination toward absenteeism. Revista de Psicodidáctica, 21, 103-119. doi: 10.1387/ RevPsicodidact.13076

Ratto, A., García, R. C., Silva, M. I., y González, M. D. C. (2015). El síndrome de quemarse por el trabajo y factores psicosociales en docentes de primaria de la ciudad de Montevideo. Ciencias Psicológicas, 9, 273-281.

Reyes-Oyola, F. A., \&y Palomino-Devia, C. (2019). Burnout syndrome, body mass index and other factors related to the practice of physical education teachers in Ibagué. Colombia. Biomédica, 39, 537-546.

Sabagh, Z., Hall, N. C., y Saroyan, A. (2018). Antecedents, correlates and consequences of faculty burnout. Educational Research, 60, 131-156.

Travers, C. (2017). Current knowledge on the nature, prevalence, sources and potential impact of teacher stress. In Educator Stress. (pp 23-54). doi: 10.1007/978-3-319-53053-6_2

Unda, S. (2010). Estudio de prevalencia del Síndrome de Quemarse por el Trabajo (SQT) y su asociación con sobrecarga y autoeficacia en maestros de primaria de la ciudad de México. Ciencia \& Trabajo, 12, 257-262.

Van Dam, A. (2016). Subgroup analysis in burnout: Relations between fatigue, anxiety, and depression. Frontiers in Psychology, 7, 90. doi:10.3389/fpsyg.2016.00090

Vela, Z. Y. (2019). Propiedades psicométricas del cuestionario para la evaluación del síndrome de quemarse por el trabajo en docentes del distrito de Juanjuí. (Tesis para obtener el título profesional de Licenciada en Psicología). Universidad Cesar Vallejo.

Villaverde, D., Unda, S., Escotto, E. A., y Flores, R. (2019). Personality traits that predict the burnout syndrome on Mexican teachers. Journal of Educational Psychology-Propositos y Representaciones, 7, 57-71. 
Viotti, S., Gil-Monte, P. R., y Converso, D. (2015). Toward validating the Italian version of the "Spanish Burnout Inventory»: a preliminary study. Revista da Escola de Enfermagem da USP, 49, 819-825. doi: 10.1590/S0080-623420150000500016

Walkey, F. H. y Green, D. E. (1992). An exhaustive examination of the replicable factor structure of the Maslach Burnout Inventory. Educational and Psychological Measurement, 52, 309-323. doi:10.1177/0013164492052002006

Wheeler D. L., Vassar M., Worley J. A., y Barnes, L. L. (2011). A reliability generalization meta-analysis of coefficient Alpha for the Maslach Burnout Inventory. Educational and Psychological Measurement, 71, 231-244. doi: 10.1177/0013164410391579

World Medical Association. (2013). Declaration of Helsinki: ethical principles for medical research involving human subjects. Journal of the American Medical Association, 310(20), 2191-2194.

Yadollahi, M., Razmjooei, A., Jamali, K., Niakan, M. H., y Ghahramani, Z. (2016). The relationship between professional quality of life (ProOol) and general health in Rajaee Trauma Hospital staff of Shiraz, Iran. Shiraz E-Medical Journal, 17, e39253. doi: $10.17795 / \operatorname{semj} 39253$ 